\title{
Comparative genome analysis of commensal segmented filamentous bacteria (SFB) from turkey and murine hosts reveals distinct metabolic features
}

\author{
Grant Hedblom \\ University of Minnesota-Twin Cities \\ Kamal Dev \\ Shoolini University

\section{Steven Bowden} \\ University of Minnesota-Twin Cities \\ David Baumler ( $\nabla$ dbaumler@umn.edu ) \\ University of Minnesota-Twin Cities
}

\section{Research Article}

Keywords: SFB, Segmented Filamentous Bacteria, turkey, comparative genomics

Posted Date: May 6th, 2021

DOI: https://doi.org/10.21203/rs.3.rs-424001/v1

License: (9) (i) This work is licensed under a Creative Commons Attribution 4.0 International License. Read Full License 


\section{Abstract}

Background: Segmented filamentous bacteria (SFB) are intestinal commensal microorganisms that have been demonstrated to induce the innate and adaptive immune responses in mouse and rat hosts. SFB are Gram-positive, sporeforming bacteria that fail to grow in vitro due to unique metabolic requirements. Recently, SFB have been implicated in improved health and growth outcomes in commercial turkey flocks. To assess the nature of SFB in turkeys and how they may differ from mammalian-associated SFB, the genome of turkey-associated SFB was compared with six representative genomes from murine hosts using an in silico approach.

Results: The SFB-turkey genome is $1.6 \mathrm{Mb}$ in length with a $\mathrm{G}+\mathrm{C}$ content of $26.14 \%$, and contains 1,604 coding sequences (CDS). Comparative genome analyses revealed that all the seven SFB strain possesses a shared set of metabolic deficiencies and auxotrophies, specifically the inability of all the SFB strains to synthesize most of the amino acids, nucleotides and cofactors, emphasizing the importance of metabolite acquisition from the host intestinal environment. Among the seven SFB genomes, the SFB-turkey genome is the largest and contains the highest number of predicted CDS. The SFB-turkey genome possesses cellular metabolism genes that are absent in the rodent SFB strains including catabolic pathways for sucrose, stachyose, raffinose and other complex glycans. Other unique genes associated with SFB-turkey genome is loci for the biosynthesis of biotin, and degradation enzymes to recycle primary bile acids, both of which may play an important role to help turkey associated SFB survive and secure mutualism with its avian host.

Conclusions: Comparative genomic analysis of seven SFB genomes revealed that each of the strains have a core set of metabolic capabilities and deficiencies that make these bacteria challenging to culture ex vivo. When compared to the murine-associated strains, turkey-associated SFB serves as a phylogenetic outgroup and a unique member among all the sequenced strains of SFB. This turkey-associated SFB strain is the first reported non-mammalian SFB genome, and highlights the impact of host specificity on the evolution of metabolic capabilities.

\section{Introduction}

The animal gastrointestinal tract harbors a diverse ecosystem of fungi, protozoans, and bacteria, many of which live in mutualistic harmony with their host. The microbial inhabitants of the intestinal mucosa provide numerous benefits to the host organism through synthesizing vitamins and cofactors, metabolizing indigestible polysaccharides and providing protection from colonization by pathogenic bacteria [1, 2]. Additionally, the succession of the gut microbiota is essential in the development and maturation of host innate immunity, impacting the overall composition of cell types present in the host epithelium and thereby alter the intestinal mucosa and the microbiome therein $[3,4]$.

Segmented filamentous bacteria (SFB) are host-adapted, intestinal symbionts that influence the adaptive and innate immune responses of their host. SFB are Gram-positive, spore-forming, microaerophilic bacteria characterized by a distinctive filamentous morphology $[5,6,7]$. SFB belong to the family Clostridiaceae and represent a unique clade of Clostridial cluster 1, also referred to as Candidatus Arthromitus, and colonize a broad range of animal hosts, including mice, rats, chickens, turkeys and humans $[7,8,9,10]$. SFB display a unique life-cycle involving intestinal binding, filamentation, differentiation, and production of either vegetative or dormant offspring [6]. SFB have been observed to bind to epithelial cells and subsequently induce cytoskeletal rearrangement within the host cell [11]. Once bound to the host epithelium, SFB grow and elongate via the formation of transverse septa to form long filaments of up to $100 \mu \mathrm{m}$ in length [12]. The ability of SFB to bind to the intestinal mucosa occurs in a host-specific manner, as attempts to inoculate germ-free hosts with SFB homogenates generated from other host species have failed [13].

SFB are one of the first studied examples of a commensal bacterial species that possesses the ability to modulate the adaptive and innate immunity of their host. In mice, the binding of SFB to the host's absorptive intestinal epithelium induces 
the differentiation of naïve CD4 + T cells to antigen-specific Th17 CD4 + cells and leads to the accumulation of Th17 cells in the terminal ileum of mice [14]. Th17 cells play a critical role in promoting and maintaining mucosal immunity through the production of several cytokines; interleukins such as IL-17A, IL-17F, IL-21, and IL-22 [15]. The production of the cytokines IL17A and IL17F aids in neutrophil chemotaxis and recruitment, while IL-22 stimulate the neutrophil-mediated production of host-defense peptides in the host epithelium, both of which lead to the clearance of bacterial or fungal pathogens from the gastrointestinal tract $[15,16]$. The induction of T-cell differentiation is likely initiated by the binding of SFB flagellins to tolllike receptor 5 (TLR5) expressed in the intestinal epithelium and subsequently initiating the NF- $K B$ pathway [17]. Intestinal colonization by SFB has been implicated in the production of secretory immunoglobulin A (slgA), which prevents the colonization of pathogenic microorganisms by blocking epithelial receptors, sterically hindering the ability of these pathogens to bind to the mucosa $[18,19]$. Additionally, mice mono-colonized with SFB displayed an increased expression of genes commonly associated with inflammation in response to colonization by pathogenic bacteria, yet displayed equal fitness to germ-free mice [16]. These findings indicate that SFB serve an essential function in the development of both the innate and adaptive immunity in mice.

SFB have been implicated in improved health and growth outcomes in commercial turkey flocks (Meleagris gallopavo). In order to investigate the microbial basis of Light Turkey Syndrome (LTS), a condition with no known causative agent that produces birds consistently below the industry standard weight, Danzeisen et al. performed 16S rRNA analysis of underweight and normal-weight flocks to determine the role of microbial succession in the development of LTS [10]. Turkey poults afflicted by LTS display symptoms of lethargy, weight loss, diarrhea, and depression early on in the growth cycle and fail to grow to their genetic potential weight, yielding birds that are 4-5 pounds lighter than the industry average [20]. While comparing the dominant operational taxonomic units (OTUs) of the higher-performing flocks to the dominant OTUs of the under-performing flocks, the higher-performing flocks harbored significantly higher proportions of SFB than their underperforming counterparts. These data suggest that SFB may serve as immunostimulatory role in the intestinal mucosa of turkey [10]. The potential of turkey-isolated SFB to serve as a novel probiotic in the commercial production of turkey makes SFB organisms of particular interest to poultry farmers and researchers, as there is an increased demand for alternatives to antibiotic use in livestock due to the growing threat of antibiotic-resistance.

As SFB have not been cultivated as a pure bacterial culture ex vivo, the ability to study the optimal growth conditions of these bacteria has not been conducted. To gain greater insights into the complex auxotrophies of turkey-associated SFB for use in future cultivation efforts, a predictive genomics and metabolomics approach was utilized to determine the genetic determinants of a turkey-associated SFB strain obtained from commercial turkey poults that has recently been sequenced [21]. In the current study, we have utilized comparative genomics to compare turkey-associated SFB [21] with mouse- and rat-associated strains of SFB previously sequenced and analyzed [17, 22, 23, 24]. Since the publication of these initial reports, which analyzed the genomes of selected strains of SFB and their metabolic characteristics, host-adaptive factors, and flagellar genes, there has been little research done to compare the characteristics of each of the represented strains and to assess the impact of host-adaptation on coevolution on the development of novel or divergent characteristics.

We identified a set of core metabolic determinants shared among all seven SFB strains and genetic traits essential for microbe-host interactions. We also determined that turkey-associated SFB are very distinct from the murine-associated strains, possessing several distinguishing metabolic characteristics including an increased capacity to degrade amino acids (serine, threonine, glutamine), metabolize a broader array of mono/disaccharide substrates, break down complex glycans, hydrolyze and recycle primary bile acids, and synthesize, rather than degrade biotin. Differences in these strains may provide further insights into the impact of coevolution with a given host organism on the development of novel characteristics.

\section{Results}

\section{General Features of the Turkey-Associated Genome}


The turkey SFB genome "Candidatus Arthromitus UMNCA01", henceforth referred to as SFB-turkey, consists of a single circular chromosome with 1,631,326 base pairs and assembled into 41 contigs (Fig. 1). The single chromosome possesses an average $\mathrm{G}+\mathrm{C}$ content of $26.14 \%$, which is similar, but slightly lower than other members of Clostridial Cluster I [22]. A putative origin of replication was identified by a distinct inflection point in coding direction at the loci of the $d n a A$ gene. A total of 1,604 coding sequences (CDS) were predicted from the SFB-turkey genome sequence, with an average length of 931 base pairs (Table 1). The genome sequence was annotated using the National Center for Biological Information (NCBI) Prokaryotic Genome Annotation Pipeline and subsequently analyzed using the Pathosystems Resource Integration Center (PATRIC) [25]. Of the predicted 1,604 CDS, 1,035 of these sequences were assigned functional roles using PATRIC, representing a $64.5 \%$ coverage of the predicted CDS, comparable with the genomes of SFB strains (Table 1). The remaining 569 CDS were not assigned functional roles and were instead designated as hypothetical proteins.

Table 1

Genome features of seven strains of Segmented Filamentous Bacteria and Clostridium beijerinckii

\begin{tabular}{|c|c|c|c|c|c|c|c|c|}
\hline $\begin{array}{l}\text { Genomic } \\
\text { features }\end{array}$ & $\begin{array}{l}\text { SFB- } \\
\text { Turkey }\end{array}$ & $\begin{array}{l}\text { SFB- } \\
\text { Mouse- } \\
\text { Japan }\end{array}$ & $\begin{array}{l}\text { SFB- } \\
\text { Mouse- } \\
\text { YIT }\end{array}$ & $\begin{array}{l}\text { SFB- } \\
\text { Mouse- } \\
\text { NYU }\end{array}$ & $\begin{array}{l}\text { SFB- } \\
\text { Mouse- } \\
\text { NL }\end{array}$ & $\begin{array}{l}\text { SFB- } \\
\text { Mouse- } \\
\text { SU }\end{array}$ & $\begin{array}{l}\text { SFB-Rat- } \\
\text { YIT }\end{array}$ & $\begin{array}{l}\text { Clostridium } \\
\text { beijerinckii } \\
\text { NRRL B- } \\
593\end{array}$ \\
\hline $\begin{array}{l}\text { Genome } \\
\text { Size (bp) }\end{array}$ & $1,631,326$ & $1,620,005$ & $1,586,397$ & $1,569,870$ & $1,654,902$ & $1,566,160$ & $1,515,556$ & $6,156,662$ \\
\hline $\begin{array}{l}\mathrm{G}+\mathrm{C} \\
\text { Content (\%) }\end{array}$ & 26.14 & 28.26 & 28.11 & 27.90 & 28.10 & 27.80 & 27.98 & 29.57 \\
\hline CDS & 1,604 & 1,540 & 1,499 & 1,503 & 1,594 & 1,511 & 1,412 & 5,808 \\
\hline tRNAs & 33 & 38 & 37 & 36 & 37 & 37 & 37 & 52 \\
\hline rRNAs & 1 & 18 & 15 & 6 & 15 & 17 & 16 & 3 \\
\hline $\begin{array}{l}\text { Hypothetical } \\
\text { Proteins }\end{array}$ & 569 & 442 & 421 & 426 & 519 & 422 & 379 & 1,854 \\
\hline $\begin{array}{l}\text { Proteins with } \\
\text { Functional } \\
\text { Assignments }\end{array}$ & 1,035 & 1,098 & 1,078 & 1,077 & 1,075 & 1,089 & 1,033 & 3,954 \\
\hline $\begin{array}{l}\text { Proteins with } \\
\text { Functional } \\
\text { Assignments } \\
\text { (\%) }\end{array}$ & $64.53 \%$ & $71.29 \%$ & $71.91 \%$ & $71.66 \%$ & $67.44 \%$ & $72.07 \%$ & $73.16 \%$ & $68.08 \%$ \\
\hline
\end{tabular}

The SFB-turkey genome contains a single rRNA operon and 33 tRNA genes, a relatively low number as compared to other members of Clostridial cluster I and among SFB representatives (Table 1) [23]. A single CRISPR sequence with 11 identical repeats and 10 unique spacer sequences was identified, along with 7 CRISPR-associated (Cas) proteins (including Cas1Cas5), which are localized on contig 38. CRISPR sequences and associated proteins confer resistance to exogenous genetic elements [26]. The presence of these CRISPR loci suggests that SFB are frequently exposed to foreign invading mobile genetic elements such as bacteriophages.

\section{Comparative Functional Genomics:}

Six representative SFB genomes from murine hosts (5 from mouse hosts and 1 from rat) were selected (GCA_000284435.1, GCA_000283555.1, GCA_000270205.1, GCA_000709435.1, GCA_000225365.2, GCA_000252785.2) to compare and assess the functional characteristics of turkey-derived SFB. The annotated genome sequences of SFB-mouse-Yit, SFB-mouseJapan, SFB-mouse-NYU, SFB-mouse-NL, SFB-mouse-SU, SFB-rat-Yit, and SFB-turkey were selected and analyzed using PATRIC analytical tools (Methods). The SFB-turkey genome was analyzed using the PATRIC similar genome finder (Methods) to find the most closely related non-SFB, free-living bacteria for use in phylogenetic comparisons. According to this analysis, Clostridium beijerinckii strain NRRL B-593 was identified as the most closely related, non-SFB, bacterial strain 
to SFB-turkey. Therefore, we used $C$. beijerinckii as a Clostridial reference genome to study the general features of murine and turkey SFB strains. Though not closely related to the murine and turkey SFB genomes, $C$. beijerinckii remained the closest relative based on the similar genome finder predictions and was therefore used as a phylogenetically related outgroup.

When comparing the genome sequence predictions of all the selected murine and turkey SFB strains, the SFB-turkey genome was predicted to possess the highest number of CDS (1604), while also possessing the second lowest number of proteins with assigned functions (1035) out of the 7 analyzed genomes of murine and turkey-SFB strains (Table 1). The SFB-turkey genome is the second largest SFB genome and is bigger than the average genome size of mouse associated SFB. SFBturkey possesses a $\mathrm{G}+\mathrm{C}$ content of $26.14 \%$, which is roughly $2 \%$ less than the murine genomes, and around $3.5 \%$ less than that of $C$. beijerinckii (Table 1). The divergence in $\mathrm{G}+\mathrm{C}$ content indicates that SFB-turkey is the most evolutionarily divergent and dissimilar strain among all of the sequenced genomes of murine and turkey-SFB strains (Fig. 1). The overall genome size of $C$. beijerinckii is approximately 3.8 times larger than the average SFB genome size and is similarly predicted to possess 3.8 times more CDS $(5,808)$ than SFB (Table 1$)$.

To assess the overall functional distribution of the analyzed SFB genomes, we compared PATRIC Subsystems assignments for each of the murine and turkey SFB strains and C. beijerinckii NRRL B-593. On average, around 25\% of all the genes (158) with subsystems assignments in SFB are involved in protein processing, while only $13 \%$ genes (224 genes) of $C$. beijerinckii genes were assigned functions that are involved in protein processing (Fig. 2, Figure S1). The genomes of analyzed SFB strains are deficient in metabolism subsystems, which comprised an average of $24 \%$ (155 genes) of the assigned SFB genes, and $43 \%$ (731 genes) of the assigned genes in C. beijerinckii. The genomes of murine-associated strains of SFB appear fairly consistent in the distribution of subsystems, while SFB-turkey possesses a greater proportion of genes associated with metabolism and a smaller proportion of genes associated with cellular processes, such as cell division, sporulation and chemotaxis, than the murine-associated strains. Within the "metabolism" category, SFB-turkey encodes more enzymes than the murine SFB strains, 169 and 155 genes respectively (Figure S1). Conversely, within the "cellular processes" category, SFB-turkey is predicted to encode fewer proteins (83) than the average of the murine SFB strains (155). Other subsystems categories, such as DNA processing, RNA processing, and energy production CDSs and associated pathways are very similarly conserved across all the murine and turkey-SFB genomes. Comparison of the subsystems assignments of SFB with $C$. beijerinckii showed that SFB have fewer genes in every subsystems category (Table S1, Figure $\mathrm{S} 1)$, but the magnitude of these differences is not consistent across all the subsystems. For example, $C$. beijerinckii is predicted to possess approximately 1.25 times more genes involved in protein processing than turkey SFB, but are predicted to possess as much as 5 times more genes involved in metabolic functions (Table S1). These results highlight that SFB strains are highly dependent on the host system and lacks very crucial pathways such as de novo synthesis of amino acids, nucleotides and cofactors. These findings serve to highlight the overall auxotrophic nature of SFB and defines their phylogenetic position between free-living bacterial and obligate intracellular symbionts [17].

In examining the phylogenetic position of SFB-turkey in relation to the murine-associated strains of SFB, it was determined that SFB-turkey is dissimilar to the murine-associated SFB strains and serves as the outgroup in a phylogenetic tree (Figure S2). All of the mouse-associated strains of SFB are phylogenetically closely related, with SFB-rat-Yit serving as the most dissimilar of the murine-associated strains of SFB. When examining the overall protein sequence similarity of each of the murine-associated strains to that of SFB-turkey, it was observed that SFB-turkey has a much lower sequence homology (avg around 70\%) than that of the murine associated strains (Fig. 3). The mouse-associated SFB strains display far greater protein sequence homology to each other (avg around 99.5\%), and are much more dissimilar to the sequences of SFB-rat-Yit and SFB-turkey (Fig. 3). These findings further underscore the role of host-association on the evolutionary divergence of SFB.

\section{Core Metabolic Characteristics of SFB genomes}


The genome of the seven analyzed SFB strains possess a core set of metabolic capabilities and deficiencies. The genome of each SFB strain exhibits several common features of anaerobic bacteria, lacking any CDS for the electron transport chain and tricarboxylic acid cycle. The genomes of SFB strains are predicted to possess all the enzymes of the glycolytic pathway for the conversion of glucose to pyruvate and the non-oxidative phase of the pentose phosphate pathway. In contrast, the enzymes glucose-6-phosphate dehydrogenase, gluconolactonase, and 6-phosphogluconate dehydrogenase of the oxidative phase of pentose phosphate pathway were absent. As expected, the genome of each SFB strain possesses pathways for the production of lactate, alcohol and acetate from pyruvate and hence, likely to generate energy through anaerobic fermentation (Fig. 4). In addition, the genomes of all the SFB-strains encode genes for alcohol, aldehyde, and lactate dehydrogenases, which facilitate the oxidation of substrates in the fermentation process. The action of pyruvate ferredoxin oxidoreductase generates acetyl-CoA, carbon dioxide and hydrogen ions; the latter is likely to be used by SFB to create a proton gradient for ATP synthesis [22]. SFB likely utilize fermentation byproducts for substrate-level phosphorylation via the action of phosphoglycerate kinase, acetate kinase, and pyruvate kinase in a method similar to that of other Clostridia [27]. Each SFB strain encodes genes for catalases and one peroxidase, indicating that these bacteria are likely to exhibit some degree of oxygen tolerance in the microaerobic environment of the intestine

SFB rely on their host and the surrounding intestinal environment for the uptake of numerous carbohydrates, as evidenced by the presence of several carbohydrate transporters. Genes encoding several phosphotransferase systems (PTSs) and ATPbinding cassette $(A B C)$ transporters for the uptake of various carbohydrates, including ascorbate, fructose, glucose, mannose, mannitol, and cellobiose were predicted from the genomes of all the SFB strains (Fig. 4). Additionally, the complete enzymatic pathways to convert these substrates to glyceraldehyde-3-phosphate were also detected. The complete enzymatic suite of the ula operon containing the genes for production of enzymes for the transport and conversion of Lascorbate to D-xylulose-5-phosphate, which is later used in the non-oxidative phase of the pentose phosphate pathway, was predicted to be encoded by the genomes of all the representative SFB strains. The genomes of all the SFB are predicted to participate in the foraging of glycans from the intestinal epithelium, encoding enzymes involved in the import and utilization of mannose, $\mathrm{N}$-acetylglucosamine, and sialic acid. To degrade these complex host glycans, SFB secrete alpha-Nacetylglucosaminidase and endo- $\beta$-N-acetylglucosaminidase. Each of the SFB strains have Sec-dependent secretory machinery; encoding for SecA, SecD, SecE, SecF, SecG, and SecY. Similar to previous analyses, the translocation-specific chaperone SecB appears to be absent in the genomes of SFB strains, suggesting that SFB utilize a modified Sec system [22].

Each of the analyzed SFB genomes is predicted to lack many critical enzymes for the biosynthesis and metabolism of amino acids, and instead likely rely on the digestion and uptake of exogenous peptides for amino acid acquisition. The SFB genomes only encode enzymes for the synthesis of lysine and for the interconversion of aspartate, glutamate, asparagine, and glutamine. Additionally, glycine hydroxymethyltransferase catalyzed interconversion reactions of glycine and serine were predicted in the genomes of all the SFB strains. Despite the lack of encoded enzymes for the production of amino acids, several permeases and transporters for the uptake of amino acids and oligopeptides are present in the genomes of SFB strain, indicating an efficient system for the uptake of amino acids from the host. To breakdown the exogenously acquired oligopeptides to utilizable amino acids, the genomes of all the SFB strain encodes numerous peptidases and proteases. The genomes of all the SFB strains either showed incomplete or completely absent biosynthetic pathways of most essential vitamins and cofactors (such as B1, B2, and B12, pyridoxine, nicotinamide, pantothenate, and biotin). Also, the genomes of SFB strains are unable to synthesize nucleotides independently; instead utilizing alternative pathways that rely largely on the exogenous uptake of nucleotide bases via the use of several PTSs and other permeases (Fig. 4).

The genomes of all the SFB strains possess complete enzymatic pathways for the shikimate shunt, the synthesis but not degradation of fatty acids, and the non-mevalonate pathway (MEP pathway) for the synthesis of terpenoids that are involved in the synthesis of peptidoglycan (Fig. 4). In order to compete with the host and members of the intestinal microbiota for iron acquisition, the genomes of SFB strains encode a number of iron transporters and uptake systems. Each 
strain encodes ATP-binding cassette proteins for the uptake of ferric iron, ferrous iron, and ferrichrome (Fig. 4). The uptake of these siderophores is essential, since all the SFB strains lack the ability to synthesize siderophores of their own.

\section{Unique Characteristics of Turkey-Associated SFB}

Despite a broad array of common characteristic features among the genome sequence of six murine and one turkeyassociated SFB, there are several unique characteristics of SFB-turkey that make this strain distinct from the murineassociated SFB strains. In considering the ability of each strain to metabolize carbohydrates, the genome of SFB-turkey is predicted to encode for many more enzymes for the uptake and utilization of a broader array of substrates. Unlike the six murine genomes, we observed that the genome of SFB-turkey is predicted to encode all the enzymes of the pentose phosphate pathway. Specifically, transaldolase is the enzyme of this pathway that is only present in the turkey-SFB genome and it catalyzes the reaction of sedoheptulose 7-phosphate and glyceraldehyde 3-phosphate to produce erythrose 4phosphate and fructose 6-phosphate (Fig. 4). The genome of SFB-turkey is also predicted to encode the fructokinase enzyme, which specifically catalyzes the transfer of a phosphate group from ATP to fructose as a first step in its utilization in glycolysis.

The host intestinal environment contains a variety of bioavailable glycans, either from the host itself or from the intestinal microflora. Like many other mucosa-associated bacteria, SFB must first cross the mucus layer that serves as a protective barrier to prevent most other bacteria from reaching the epithelium. It has been previously reported that SFB's specialized ability to utilize certain glycan residues, such as mannose and $\mathrm{N}$-acetylglucosamine, provide a competitive advantage in intestinal colonization [23]. Unlike the murine-associated strains of SFB, SFB-turkey is predicted to utilize sucrose, raffinose, stachyose, and sialic acid as potential carbohydrate sources. SFB-turkey is also predicted to encode the enzyme betafructosidase (invertase), which catalyzes the hydrolysis of terminal non-reducing beta-D-fructofuranoside residues leading to the conversion of sucrose to D-fructose and D-glucose, raffinose to melibiose and D-fructose, and stachyose to manninotriose and D-fructose (Fig. 4). To import these carbohydrates, only the SFB-turkey genome encodes a putative phosphotransferase system for the uptake of sucrose and the maltodextrin $\mathrm{ABC}$ transporter $(\mathrm{MsmX})$. The specific presence of these enzymes in the SFB-turkey genome suggests that it has developed a competitive advantage in comparison to murine SFB to adapt to a highly complex host intestinal environment in order to utilize intermediate glycans as primary carbohydrate sources.

In considering the ability of each strain to synthesize and utilize purines and pyrimidines, SFB-turkey is distinct from the murine-associated SFB strains. The genome of SFB-turkey encodes for the metabolic pathway for the de novo synthesis of purine nucleotides from phosphoribosyl pyrophosphate (PRPP) through the action of uracil phosphoribosyltransferase, suggesting that turkey-associated SFB alone utilize pentose phosphate pathway products in the biosynthesis of nucleotides (Fig. 4, Figure S3) [28]. The genome of SFB-turkey also possesses a pathway for the conversion of L-glutamine to orotidine5-phosphate, which is involved in the aforementioned pathway for the synthesis of nucleotides from PRPP. This pathway represents the only example in the examined SFB genomes that utilizes amino acids as an intermediate for the synthesis of nucleotides and may underscore an evolutionary divergence node between SFB-turkey and the murine SFB strains.

In addition to the aforementioned differences in carbohydrate and nucleotide metabolism, SFB-turkey is the only SFB strain predicted to be able to synthesize biotin. SFB-turkey has been predicted to encode 8-amino-7-oxononanoate, adenosylmethionine-8-amino-7-oxononanoate transaminase, dethiobiotin synthase and biotin synthase, all of which allow for the biosynthesis of biotin. In contrast, murine-associated SFB strains are predicted to lack all the enzymes involved in synthesis of biotin. To compensate for the lack of biosynthetic pathways for the production of biotin, murine-associated strains are predicted to encode the BioY biotin ECF transporter, which is likely to be involved in the import of biotin into the cell and its subsequent metabolism. Interestingly, SFB-turkey genome is also the only analyzed strain not predicted to encode the BioY biotin ECF transporter. Each SFB strain is predicted to encode all the subunits of the biotin-ligase holoenzyme for the conversion of biotin into biotinyl-CoA and holocarboxylase, which are involved in the production of urea and fatty acid biosynthesis respectively. 
Another major difference between the genomes is that SFB-turkey possesses genes that encode enzymes for the hydrolysis of primary bile acids that are absent from the murine SFB genomes. SFB-turkey is predicted to encode choloylglycine hydrolase (bile acid hydrolase), which allows for the production of taurine, cholate, glycine and chenodeoxycholate from the conjugated bile salts. With the exception of glycine, SFB-turkey has no apparent enzymatic steps or pathways to utilize these secondary bile acids and their precursors. Though this enzyme was previously predicted to be encoded by SFB-mouseSU [24], our analysis predicted it to be only encoded by SFB-turkey. Bile acids have been reported as immunomodulatory and possess antimicrobial activities [29], and this may require SFB-turkey to synthesize bile acid hydrolase to overcome the antimicrobial effects of these host factors in the avian ileum. This mechanism has been reported in other gut commensals [30]. Deconjugated bile salts are less efficiently absorbed and more likely to be excreted by the host, increasing the demand for cholesterol for de novo synthesis of bile acids to replenish the loss of bile salts [31]. In mouse studies, comparing mice inoculated with strains of Bacteroides with and without bile salt hydrolases, it was found that the mice colonized with bile salt hydrolase-deficient bacteria gained less weight and had lower levels of fats and cholesterol in their blood and liver and tend to metabolize fats rather than carbohydrates to generate energy [32].

Finally, SFB-turkey was the only SFB strain predicted to encode the subunits of a phosphotransferase system for the import and phosphorylation of $\mathrm{N}$-acetylmuramic acid and $\mathrm{N}$-acetylmuramic acid-6 phosphate etherase for the conversion of $N$ acetylmuramic acid-6 phosphate to N-acetylglucosamine-6-phosphate (Fig. 4). N-acetylglucosamine-6-phosphate is used as an intermediate in the production of the host peptidoglycan layer and allows SFB-turkey to utilize alternate routes to synthesize the cell wall of these bacteria.

\section{Genes Involved in Sporulation and Motility}

According to the PATRIC subsystem assignments of each of the SFB genomes, each of the examined SFB strains encodes several enzymes involved in sporulation and germination. Key genes encoding sporulation-related sigma factors were identified in each of the SFB strains. Like many other closely related Clostridia, each SFB strain lacks the essential enzymes involved in the phosphorelay system for sporulation initiation, such as Kin, Spo0B, and Spo0F [22]. The genomes of all the SFB strains are predicted to encode the same set of sporulation genes, with the exception of SpollR, a gene required for processing and compartmentalization of pro-SigE, which is not encoded by SFB-turkey. The genomes of all the murine and turkey-SFB encodes a single ger operon, which contains germinant receptors belonging to the GerA family. In the genome of each SFB-strain, the ger operon is directly preceded by $c p h A B$ and ispE genes upstream of the operon. The $c p h A B$ genes encode for cyanophycin synthase and cyanophycinase, two enzymes necessary for the biosynthesis of cyanophycin, an amino acid polymer comprised of Aspartic acid and Arginine [22]. Cyanophycin may be used by germinating SFB spores as an amino acid source during the germination process. The ispE gene encodes 4-diphosphocytidyl-2-C-methyl-D-erythritol kinase, which is a necessary enzyme in the methyl-erythritol phosphate (MEP) pathway for the synthesis of terpenoids [22]. Terpenoids are essential components in peptidoglycan and cell membrane synthesis. The proximity of these gene loci to the ger operon suggests that the synthesis of these enzymes may be coupled with germination and transcribed in tandem with the operon.

The genomes of the murine-associated SFB strains encode 32 genes for the synthesis and assembly of flagella, whilst the turkey-associated SFB is predicted to encode only 29 of such genes. The flagella genes include several encoding the flagellin subunit FliC, as previously indicated in other studies examining the genome sequences of SFB strains [17, 22]. Each SFB strain encodes at least two copies of the fliC gene, with SFB-turkey encoding 4 copies. Flagellins are key filament proteins that have been implicated in the innate production of Th17 cells, serving as an agonist of Toll-like receptor 5 (TLR5) in intestinal dendritic cells and triggering the NF- $K B$ signaling pathway of cytokine production [33, 34]. The presence of a complete flagellar apparatus in most SFB strains suggests that these organisms may be motile at some point in the SFB lifecycle. SFB may also use flagella for penetrating the intestinal mucosa in a method similar to that of other intestinal bacteria [35]. Despite the proposed function of flagella in cellular motility, flagellated SFB cells have yet to be observed microscopically. Unlike the murine-associated strains of SFB, the SFB-turkey genome lacks the genes encoding FliL, FlgN, and FlbD, which are responsible for flagellar rotation, flagellar assembly initiation, and transcriptional regulation, respectively 
[36]. The absence of these aforementioned genes may indicate that SFB-turkey is the only strain with an incomplete flagellar apparatus and perhaps lost the ability to utilize this structure for motility and chemotaxis related functions. The flagella in SFB-turkey may only serve as a surface antigen to present to TLR5 in the intestinal epithelia, losing the functionality of the complete flagellar apparatus during evolution. However, the perceived absence of these genes in the SFB-turkey genome may be due to low sequence homology of predicted CDS to known proteins. Finally, each SFB strain is predicted to encode 8 chemotaxis related proteins, including $\mathrm{CheY}$ which is involved in transmitting chemoreceptor signals directly to the flagellar motor complex [36].

\section{Discussion}

The whole-genome sequence of SFB-turkey and subsequent comparison of this strain to several murine SFB strains, revealed a number of conserved characteristics of all segmented filamentous bacteria, and several metabolic capabilities that are unique to SFB-turkey. All SFB have a highly reduced genome size in comparison to other free-living bacterial genomes and are deficient in pathways for the de novo biosynthesis of amino acids, cofactors and nucleotides, underscoring the auxotrophic nature and non-culturability of this unique group of Clostridia. To counteract these metabolic deficiencies, each SFB strain encodes numerous proteases, peptidases, permeases, and transporters to aid in the digestion and uptake of exogenous amino acids and nucleotides that are present in the host intestinal epithelia, capitalizing on the bioavailability of host or microbiome factors for survival and functionality. Due to the absence of core metabolic pathways, all the SFB strains are not tractable to culture under laboratory conditions. Such genome analysis could help in providing insights in the development of future experimental cultures.

SFB thrive in the host intestinal epithelium, an ecological niche rich in simple carbohydrates produced by the degradation of complex carbohydrates by brush-border digestive enzymes. SFB likely require these smaller monosaccharides and disaccharides to survive, as they lack the enzymes for the digestion of more complex glycans and polysaccharides. However, unlike the murine-associated strains of SFB, turkey-associated SFB is predicted to encode a much more diverse set of enzymes for the degradation of carbohydrates, including invertase, which allows this strain to utilize sucrose, raffinose, and stachyose as potential carbohydrate sources. The ability of SFB-turkey to utilize a broader array of carbohydrates and glycans than their murine-associated counterparts may indicate an evolutionary divergence due to the dietary difference and intestinal microbiome compositions of commercial turkey in comparison to mice or rats. Additionally, turkey-associated SFB utilize amino acids and nucleotides in a manner distinct to that of each of the murine-associated strains. Unlike the murineassociated strains of SFB, turkey-associated SFB are predicted to be able to synthesize biotin, circumventing a key metabolic deficiency of its murine-associated counterparts. SFB-turkey was the only strain predicted to encode choloylglycine hydrolase to deconjugate primary bile acids, allowing the SFB-turkey strain to avoid the antimicrobial action of these compounds and may potentially serve to benefit the avian host through lowering host cholesterol, warranting further study and examination. These observed divergences in metabolic capabilities suggest that each member of this group of bacteria has developed host-adapted capabilities to best associate with their host and the gut microbiome therein, securing mutualism and survival. In addition to the immunostimulatory effects of SFB on the stimulation of Th17 cell development and immunoglobulin production, the presence of choloylglycine hydrolase may also serve to improve performance outcomes of commercial flocks in the prevention of Light Turkey Syndrome.

In conclusion, our work provides important evidence suggesting that segmented filamentous bacteria isolated from evolutionarily divergent host organisms possess distinct enzymatic pathways for carbohydrate, nucleotide, amino acid, and vitamin and cofactor metabolism, indicating the role of host-adaptation in securing a mutualistic relationship with their host. The core metabolic deficiencies of each SFB strain highlighted in our study will aid in future attempts to cultivate these bacteria from other host organisms.

\section{Materials And Methods}




\section{Genome Sequencing and Assembly of SFB-turkey}

Candidatus Arthromitus UMNCA01 strain of SFB was isolated from the Gut Microbiome of commercial turkey raised in a research flock at the University of Minnesota. The UMNCA01 genome can be accessed via the GenBank accession number GCA_001655775.1. The genome sequence and global statistics of UMNCA01 genome have been recently published by Hedblom et al. [21].

\section{Genome of SFB Bacteria Used in Current Study}

The strains used in this study represent assembled SFB genomes of mouse, rat and turkey hosts are as follows: SFB-mouseYIT (GCA_000284435.1), SFB-rat-YIT (GCA_000283555.1), SFB-mouse-Japan (GCA_000270205.1), SFB-mouse-NL (GCA_000709435.1), SFB-mouse-NYU (GCA_000225365.2), SFB-mouse-SU (GCA_000252785.2), and SFB-turkey (GCA_001655775.1).

\section{Assessing Functional Similarity of SFB genomes}

The genomes of seven murine and turkey SFB were compared and analyzed at the level of genes and protein sequences using the Pathosystems Resource Integration Center (PATRIC) Bioinformatics Resource Center [25]. To compare the functional distribution of the contents of the SFB genomes, we used the PATRIC Subsystems analysis tool. Subsystems, like clusters of orthologous genes are collections of functionally related proteins that are enumerated and divided into superclass (example: metabolism), class (example: biotin metabolism), subclass, and subsystem [37, 38]. In order to enable comparative analyses of eight genomes and original annotations using consistent vocabulary, all of the genomes were annotated using a customized version of the RAST tool kit (RASTtk) through the PATRIC Genome Annotation service [39]. PATRIC assigns protein encoding genes protein family membership in order to drive comparative analysis tools and KEGG metabolic pathway information [40].

\section{Detection of Differential Metabolic Pathways Among Genomes of SFB}

In order to compare the presence and absence of proteins and specific metabolic pathways, the PATRIC Proteome Comparison tool, which uses a bidirectional best BLASTP analysis, was implemented [41]. In order to sort and compare these protein families, PATRIC Cross-Genus Protein Families (PGfams) were used [42]. PGfams are comparable clusters of proteins that likely have been assigned similar functional assignments and annotations. For proteins with no annotation, BLAST similarity search was used to cluster these sequences [41]. The PGfams clusters were used for cross-genus comparison due to their slightly relaxed clustering criteria, which was selected for the relative novelty and scarcity of SFB genomes. The Proteome Comparison tool allowed for the filtering of functionally assigned gene families based on the presence or absence of these genes across the examined genomes. This tool was used to examine functional families that were specifically present or absent only in SFB-turkey.

\section{Sequence Similarity and Phylogenetic Tree Construction}

To determine the sequence similarity and phylogenetic relationship between the genomes of SFB, the PATRIC Similar Genome Finder was implemented [25]. The Similar Genome Finder utilizes Mash, which functions by reducing whole genome sequences into representative sketches that are used for estimating mutation rates of analyzed k-mer sequences [43]. This tool compares against all public genome sequences and yields the distance between these sequences and a corresponding $P$-value [44]. For phylogenetic tree construction, the genomes of SFB strains were analyzed through the PATRIC Codon Trees pipeline, which utilizes PATRIC Cross-Genus Protein Families (PGfams) to align 1000 single copy protein and nucleotide sequences using MUSCLE and Biopython respectively $[45,46]$. Support values for the phylogenetic tree were generated from 100 rounds of rapid bootstrapping in RAxML [47]. The $P$-value threshold used was 0.001 , and the maximum distance between sequences was set at 0.5 to allow for SFB-rat and SFB-turkey to be included.

\section{Abbreviations}


SFB: Segmented filamentous bacteria; CDS: coding sequences; PATRIC: Pathosystems Resource Integration Center; CRISPR: Clustered regularly interspaced palindromic repeats; PTS: phosphotransferase system; ABC: ATP-binding cassette; TLR5:

Toll-like receptor 5; BLAST: Basic Local Alignment Search Tool; LTS: Light Turkey Syndrome;

\section{Declarations}

Acknowledgements

The authors are thankful to the Department of Food Science and Nutrition at the University of Minnesota for providing the facilities and assistance for carrying out this study.

Authors' contributions

$\mathrm{GH}$ and DB conceived and designed the study. The analysis of the data was carried out by $\mathrm{GH}$. The manuscript was drafted by $\mathrm{GH}$ and $\mathrm{DB}$ and reviewed by $\mathrm{KD}$ and $\mathrm{SB}$. All authors read, commented and approved the final manuscript.

Funding

We would like to thank USDA-AFRI award \#2016-67015-24911, University of Minnesota's Global Food Ventures program, and the University of Minnesota's Healthy Foods, Healthy Lives institute.

Availability of data and materials

The datasets utilized in the present study are available in the National Center for Biology Information (NCBI) [https://www.ncbi.nlm.nih.gov/], GenBank repository, under accession numbers as GCA_000284435.1, GCA_000283555.1, GCA_000270205.1, GCA_000709435.1, GCA_000225365.2, GCA_000252785.2, and GCA_001655775.1. Additional results supporting findings presented in this study are provided in the supplementary information section.

Ethics approval and consent to participate

Not applicable

Consent for publication

Not applicable

Competing interests

The authors declare that they have no competing interests.

\section{References}

1. Backhed F, Ley RE, Sonnenburg JL, Peterson DA, Gordon JI. Host-bacterial mutualism in the human intestine. Science. 2005;307:1915-20.

2. Hooper LV, Midtvedt T, Gordon Jl. How host-microbial interactions shape the nutrient environment of the mammalian intestine. Ann Rev Nutr. 2002;22:283-307.

3. Atarashi K, Umesaki Y, Honda K. Microbiotal influence on T cell subset development. Semin Immunol. 2011;23:146153.

4. Ivanov II, Littman DR. Modulation of immune homeostasis by commensal bacteria. Curr Opin Microbiol. 2011;14:106114.

Page $11 / 16$ 
5. Ericsson AC, Hagan CE, Davis DJ, Franklin CL. Segmented filamentous bacteria: commensal microbes with potential effects on research. Comp Med. 2014;64:90-98.

6. Schnupf P, Gaboriau-Routhiau V, Cerf-Bensussan N. Host interactions with segmented filamentous bacteria: an unusual trade-off that drives the post-natal maturation of the gut immune system. Semin Immunol. 2013;25:342-351.

7. Hedblom GA, Reiland HA, Sylte MJ, Johnson TJ, Baumler DJ. Segmented Filamentous Bacteria - Metabolism Meets Immunity. Front Microbiol. 2018;9:1991.

8. Klaasen HL, Koopman JP, Van den Brink ME, Bakker MH, Poelma FG, Beynen AC. Intestinal, segmented, filamentous bacteria in a wide range of vertebrate species. Lab Anim. 1993;27:141-150.

9. Snel J, Heinen PP, Blok HJ, Carman RJ, Duncan AJ, Allen PC, Collins MD. Comparison of 16S rRNA sequences of segmented filamentous bacteria isolated from mice, rats, and chickens and proposal of "Candidatus Arthromitus". Int $J$ Syst Bacteriol. 1995;45:780-782.

10. Danzeisen JL, Calvert AJ, Noll SL, McComb B, Sherwood JS, Logue CM, et al. Succession of the turkey gastrointestinal bacterial microbiome related to weight gain. PeerJ. 2013;1:e237.

11. Blumershine RV, Savage DC. Filamentous microbes indigenous to the murine small bowel: a scanning electron microscopic study of their morphology and attachment to the epithelium. Microb Ecol. 1978;4:95-103.

12. Chase DG, Erlandsen SL. Evidence for a complex life cycle and endospore formation in the attached, filamentous, segmented bacterium from murine ileum. J Bacteriol. 1976;127:572-583.

13. Tannock GW, Miller JR, Savage DC. Host specificity of filamentous, segmented microorganisms adherent to the small bowel epithelium in mice and rats. Appl Environ Microbiol. 1984;47:441-442.

14. Farkas AM, Panea C, Goto Y, Nakato G, Galan-Diez M, Narushima S, et al. Induction of Th17 cells by segmented filamentous bacteria in the murine intestine. J Immunol Methods. 2015;421:104-111.

15. Schnupf P, Gaboriau-Routhiau V, Sansonetti PJ, Cerf-Bensussan N. Segmented filamentous bacteria, Th17 inducers and helpers in a hostile world. Curr Opin Microbiol. 2017;35:100-109.

16. Ivanov I, Atarashi K, Manel N, Brodie EL, Shima T, Karaoz U, et al. Induction of intestinal Th17 cells by segmented filamentous bacteria. Cell. 2009;139,485-498.

17. Kuwahara T, Ogura Y, Oshima K, Kurokawa K, Ooka T, Hirakawa H, et al. The lifestyle of the segmented filamentous bacterium: a non-culturable gut-associated immunostimulating microbe inferred by whole-genome sequencing. DNA Res. 2011;18,291-303.

18. Talham GL, Jiang HQ, Bos NA, Cebra JJ. Segmented filamentous bacteria are potent stimuli of a physiologically normal state of the murine gut mucosal immune system. Infect Immun. 1999;67:1992-2000

19. Mantis NJ, Rol N, Corthésy B. Secretory IgA's complex roles in immunity and mucosal homeostasis in the gut. Mucosal Immunol. 2011;4(6):603-11.

20. Mor SK, Sharafeldin TA, Abin M, Kromm M, Porter RE, Goyal SM, et al. The occurrence of enteric viruses in light turkey syndrome. Avian. Pathol. 2013;42,497-501.

21. Hedblom GA, Dev K, Bowden SD, Weber B, Noll S, Baumler DJ, Johnson TJ. Draft genome sequence of "Candidatus Arthromitus" UMNCA01, a suspected commensal isolated from the gut microbiome of commercial turkey. Microbiol Resour Announc. 2020;9,4:e01143-19.

22. Prakash T, Oshima K, Morita H, Fukuda S, Imaoka A, Kumar N, et al. Complete genome sequences of rat and mouse segmented filamentous bacteria, a potent inducer of Th17 cell differentiation. Cell Host Microbe 2011;10:273-284.

23. Sczesnak A, Segata N, Qin X, Gevers D, Petrosino JF, Huttenhower C, et al. The genome of Th17 cell-inducing segmented filamentous bacteria reveals extensive auxotrophy and adaptations to the intestinal environment. Cell Host Microbe. 2011;10:260-272.

24. Pamp SJ, Harrington ED, Quake SR, Relman DA, Blainey PC. Single-cell sequencing provides clues about the host interactions of segmented filamentous bacteria (SFB). Genome Res. 2012;22:1107-1119.

Page $12 / 16$ 
25. Wattam AR, Davis JJ, Assaf R, Boisvert S, Brettin T, Bun C, et al. Improvements to PATRIC, the all-bacterial Bioinformatics Database and Analysis Resource Center. Nucleic Acids Res. 2017;4(45):535-542.

26. Horvath P, and Barrangou R. CRISPR/Cas, the immune system of bacteria and archaea. Science. 2010;327:167-170.

27. Desvaux M,. Clostridium cellulolyticum: model organism of mesophilic cellulolytic clostridia, FEMS Microbiol Rev. 2005;29:741-64.

28. Selwood T, Jaffe E. Dynamic dissociating homo-oligomers and the control of protein function. Arch Biochem Biophys. 2012;519(2):131-43.

29. Inagaki T, Moschetta A, Lee YK, Peng L, Zhao G, Downes M, et al. Regulation of antibacterial defense in the small intestine by the nuclear bile acid receptor. Proc Natl Acad Sci 2006;103:3920-3925.

30. Jones BV, Begley M, Hill C, Gahan CG, Marchesi JR. Functional and comparative metagenomic analysis of bile salt hydrolase activity in the human gut microbiome. Proc Natl Acad Sci. 2008;105:13580-13585.

31. Hofmann AF. The function of bile salts in fat absorption. The solvent properties of dilute micellar solutions of conjugated bile salts. Biochem J. 1963;89:57-68.

32. Yao L, Seaton SC, Ndousse-Fetter S, Adhikari AA, DiBenedetto N, Mina Al, et al. A selective gut bacterial bile salt hydrolase alters host metabolism. eLife. 2018;7:e37182.

33. Hayashi F, Smith KD, Ozinsky A, Hawn TR, Yi EC, Goodlett DR, et al. The innate immune response to bacterial flagellin is mediated by Toll-like receptor 5. Nature. 2001;410:1099-1103.

34. Uematsu S, Akira S. Immune responses of TLR5 + lamina propria dendritic cells in enterobacterial infection. J Gastroenterol. 2009;44:803-811.

35. Guerry P. Campylobacter flagella: not just for motility. Trends Microbiol. 2007;15:456-461.

36. DeRosier D. The Turn of the Screw: The Bacterial Motor Protein. Cell. 1998;93:17-20.

37. Overbeek R, Begley T, Butler RM, Choudhuri JV, Chuang HY, Cohoon M, et al. The subsystems approach to genome annotation and its use in the project to annotate 1000 genomes. Nucleic Acids Res. 2005;33:5691-5702.

38. Overbeek R, Olson R, Pusch GD, Olsen GJ, Davis JJ, Disz T, et al. The SEED and the Rapid Annotation of microbial genomes using Subsystems Technology (RAST). Nucleic Acids Res. 2013;42:206-214.

39. Brettin T, Davis JJ, Disz T, Edwards RA, Gerdes S, Olsen GJ, et al. RASTtk: a modular and extensible implementation of the RAST algorithm for building custom annotation pipelines and annotating batches of genomes. Sci Rep. 2015;5:8365.

40. Kanehisa M, Sato Y, Kawashima M, Furumichi M, Tanabe M. KEGG as a reference resource for gene and protein annotation. Nucleic Acids Res. 2016;44:457-462.

41. Johnson M, Zaretskaya I, Raytselis Y, Merezhuk Y, McGinnis S, Madden TL. NCBI BLAST: a better web interface. Nucleic Acids Res. 2008;36:W5-W9.

42. Davis JJ, Gerdes S, Olsen GJ, Olson R, Pusch GD, Shukla M, et al. PATtyFams: protein families for the microbial genomes in the PATRIC Database. Front Microbiol. 2016;7:118.

43. Ondov BD, Treangen TJ, Melsted P, Mallonee AB, Bergman NH, Koren S, et al. Mash: fast genome and metagenome distance estimation using MinHash. Genome Biol. 2016;17:132

44. Davis JJ, Wattam AR, Aziz RK, Brettin T, Butler R, Butler RM, et al. The PATRIC Bioinformatics Resource Center: expanding data and analysis capabilities. Nucleic Acids Research. 2019;10:943.

45. Edgar RC. MUSCLE: multiple sequence alignment with high accuracy and high throughput. Nucleic Acids Res. 2004;32:1792-1797.

46. Cock PJ, Antao T, Chang JT, Chapman BA, Cox CJ, Dalke A, et al. Biopython: freely available Python tools for computational molecular biology and bioinformatics. Bioinformatics. 2009;25:1422-1423. 
47. Stamatakis A, Hoover P, Rougemont J. A rapid bootstrap algorithm for the RAxML web servers. Syst Biol. 2008;57:758771.

\section{Figures}

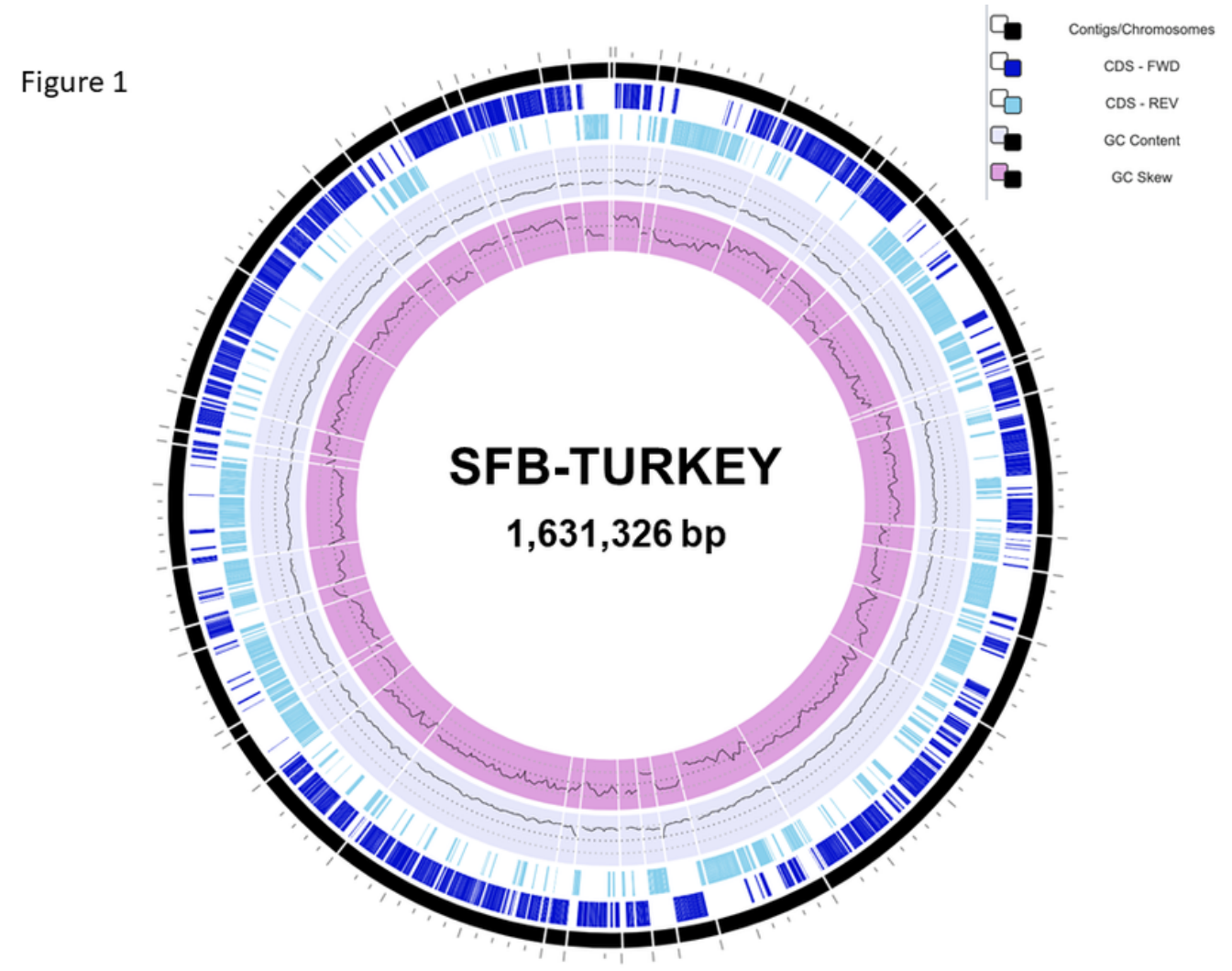

Figure 1

Circular map of the SFB-Turkey Genome The 41 contigs were arranged into a circular pseudochromosome (see Methods). Circles from the outside in are as follows: (1) Contig mapping including assembly gaps. (2) Coding Sequences (CDS) on the forward strand of the chromosome. (3) Coding Sequences (CDS) on the reverse strand of the chromosome. (4) $G+C$ content of each loci on the bacterial chromosome. (5) G+C skew at each loci on the bacterial chromosome. 
Figure 2

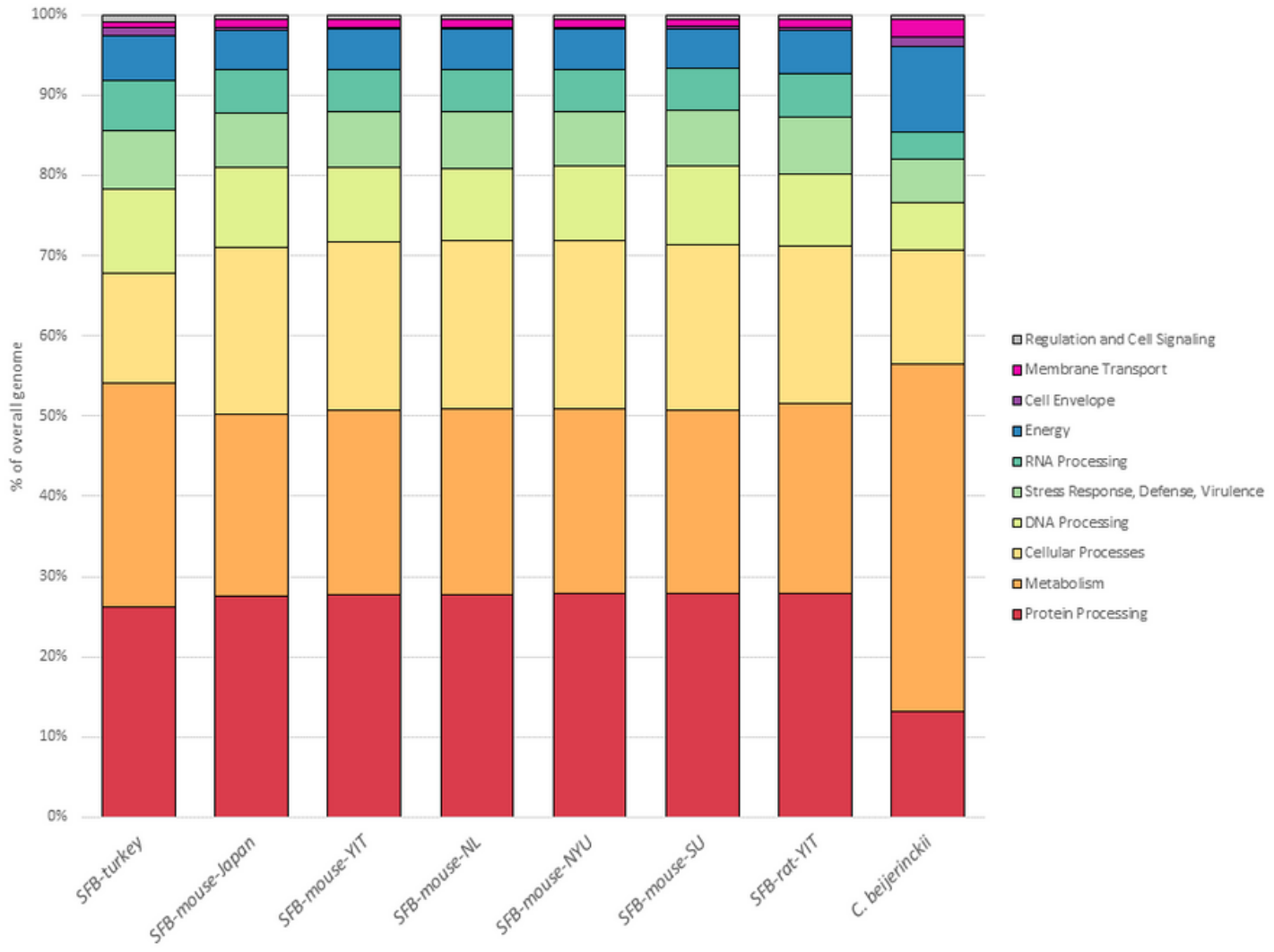

Figure 2

Subsystems Assignments of seven strains of segmented filamentous bacteria and Clostridium beijerinckii Overview of the PATRIC Subsystem assignments of the seven analyzed genomes of SFB strains and Clostridium beijerinckii as represented by the percent of genes in each Subsystem category.

Figure 3

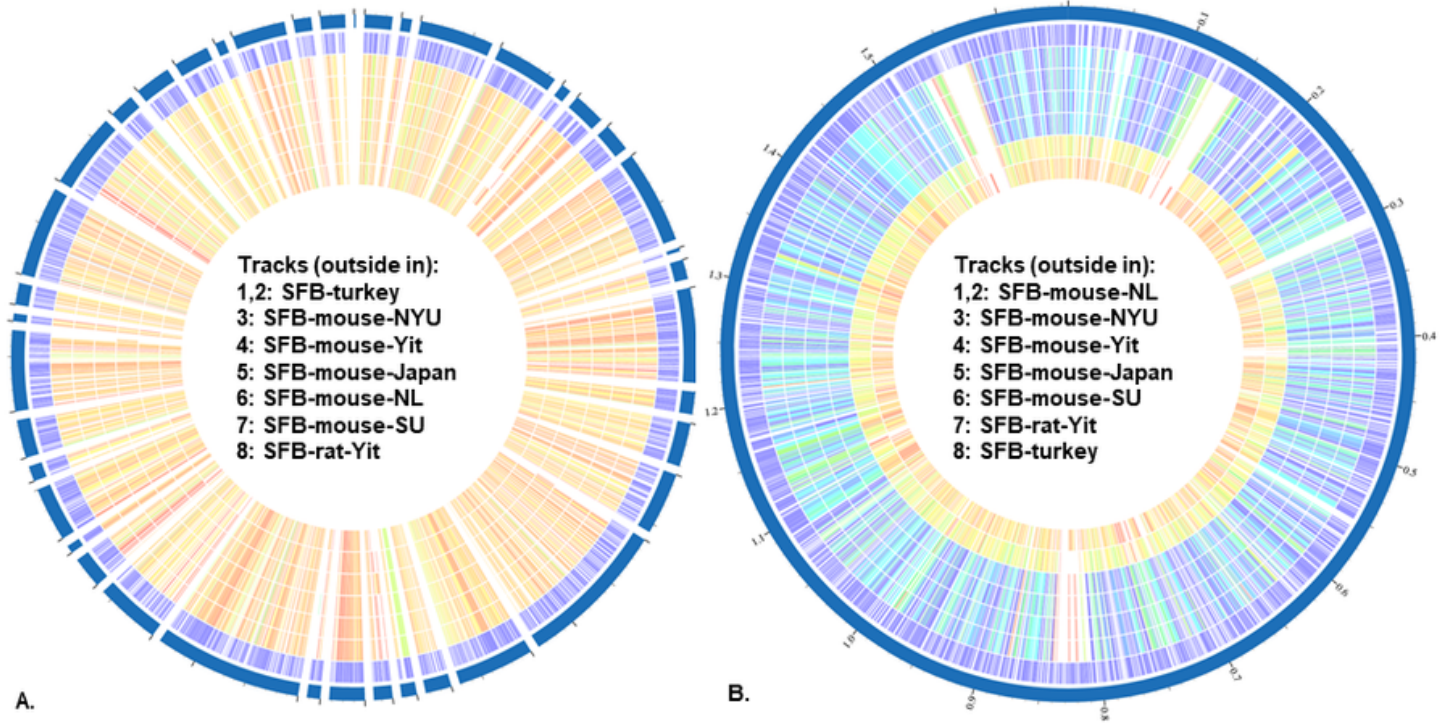

Percent protein sequence identity

\begin{tabular}{|c|c|c|c|c|c|c|c|c|c|c|c|c|c|c|c|c|}
\hline & \multicolumn{16}{|c|}{ n sequence Id } \\
\hline Bidirectional best hit & 100 & 99.9 & 99.8 & 99.5 & 99 & 98 & 95 & 90 & 80 & 70 & 60 & 50 & 40 & 30 & 20 & 10 \\
\hline Unidirectional best hit & 100 & 99.9 & 99.8 & 99.5 & 99 & 98 & 95 & 90 & 80 & 70 & 60 & 50 & 40 & 30 & 20 & 10 \\
\hline
\end{tabular}


Figure 3

Proteome Comparison of seven strains of segmented filamentous bacteria Protein sequence-based genome comparison of genomes of seven SFB genomes using bidirectional BLASTP using SFB-turkey as a reference genome (A), or SFB-mouse-NL as a reference genome $(B)$.

Figure 4

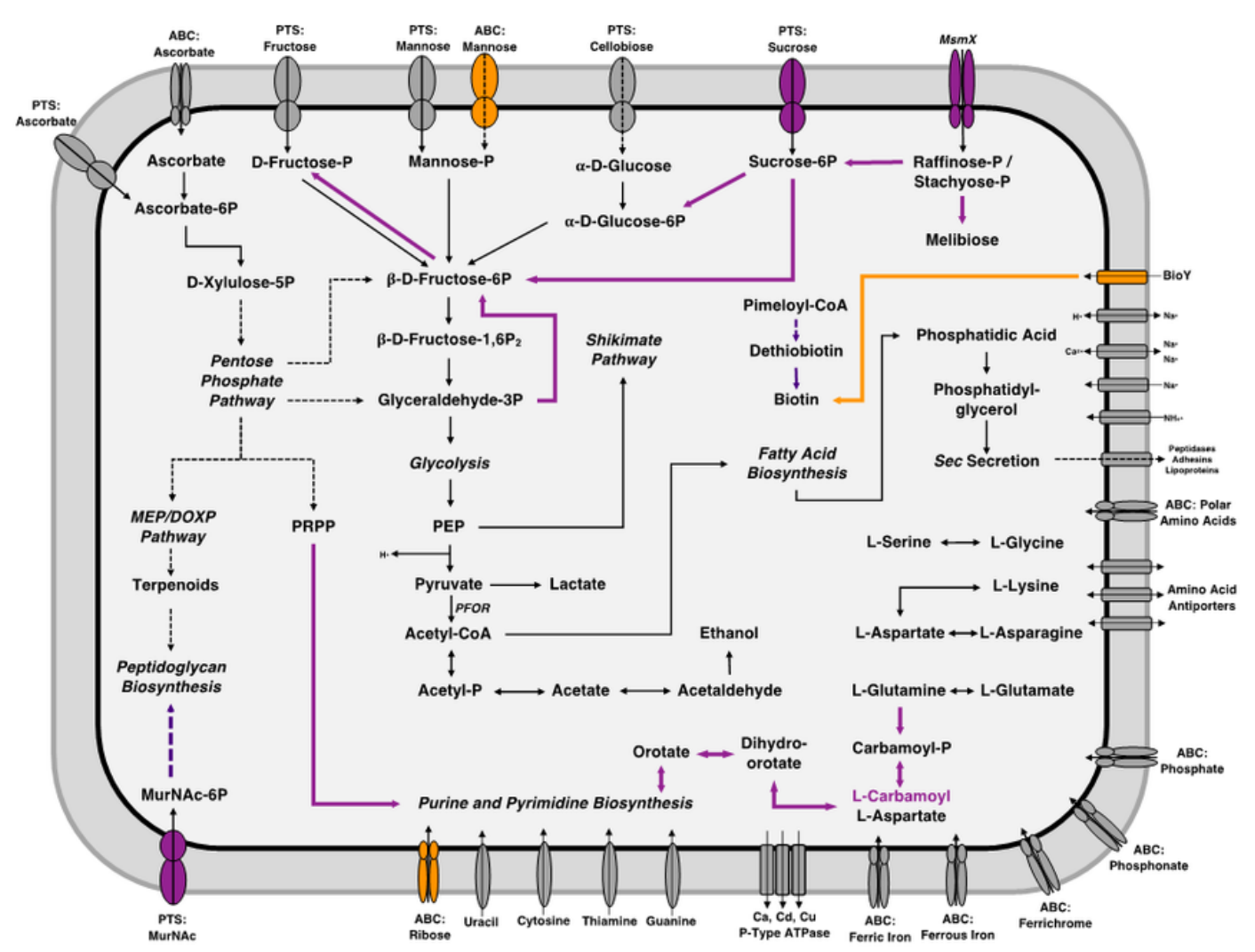

Figure 4

Comparative Metabolic Pathways Between SFB-turkey and Murine SFB Strains A schematic comparison of the metabolic capabilities of SFB strains isolated from turkey and murine hosts. Color coding for divergent metabolism is as follows: enzymatic pathways and transporters that are shared by all SFB strains appear in black, complete metabolic pathways conserved in each SFB strain appear in bold and italics, enzymatic pathways and transporters that are unique to SFB-turkey appear in purple, enzymatic pathways and transporters that are unique to the murine-associated strains appear in orange. Oval shapes are used to indicate permeases, rectangles represent import/export-type transporters. Contiguous lines represent single enzymatic reactions, while dashed lined represent a series of conserved enzymatic pathways.

\section{Supplementary Files}

This is a list of supplementary files associated with this preprint. Click to download.

- FigureS1.pptx

- Figures2.pptx

- FigureS3.pptx

- Supplementaltableandfigurelegends.docx

- Tables1.pptx 